\title{
Nucleus of the Spinal Tract of the Trigeminal Nerve
}

National Cancer Institute

\section{Source}

National Cancer Institute. Nucleus of the Spinal Tract of the Trigeminal Nerve. NCI

Thesaurus. Code C33188.

The site of termination for fibers of the spinal trigeminal tract, located in the lateral medulla and extending in into the caudal pons, that receives input from the ipsilateral face via somatic afferent fibers for touch, pain, and temperature. 